\title{
An Anthropomorphic AR-Based Personal Information Manager and Guide
}

\author{
Andreas Schmeil and Wolfgang Broll \\ Fraunhofer FIT, Collaborative Virtual and Augmented Environments Department, \\ Schloss Birlinghoven, 53754 Sankt Augustin, Germany \\ Andreas.Schmeil@googlemail.com, Wolfgang.Brollafit.fraunhofer.de
}

\begin{abstract}
The use of personal electronic equipment has significantly increased during recent years. Augmented Reality (AR) technology enables mobile devices to provide a very rich user experience by combining mobile computing with connectivity and location-awareness. In this paper we discuss the approach and development of an Augmented Reality-based personal assistant, combining the familiar interface of a human person with the functionality of a locationaware digital information system. The paper discusses the main components of the system, including the anthropomorphic user interface as well as the results of an initial prototype evaluation.
\end{abstract}

Keywords: Augmented Reality, Mobile Computing, Virtual Humans, Anthropomorphic User Interfaces, Digital Assistants, Environment Model, Location Based Systems.

\section{Introduction}

Mobile electronic devices are being used more and more in every day life. Mobile phones, PDAs and laptop computers have replaced more traditional media and tools. Thus many people use laptop computers instead of desktop PCs today, and PDAs and smartphones have replaced traditional paper-based organizers. Another development we can observe today is the increasing availability of location-based services. In recent years mobile providers have started providing navigation and other cell based services. The popularity of such services has also increased with the introduction of new connectivity technologies such as WiFi, WiMax and UMTS. However, most available devices and systems share these problems:

- Compared to having a real assistant, many tasks are very cumbersome and timeconsuming, due to the limited user interface provided by the devices.

- The range of applications and services are limited, due to limits of the existing devices and displays used in them.

- Existing devices are difficult to use if you want to use your hands for another task or if you need them free.

- Users do not benefit appropriately from available location information, as it is mostly not integrated with the organizer functionality. 


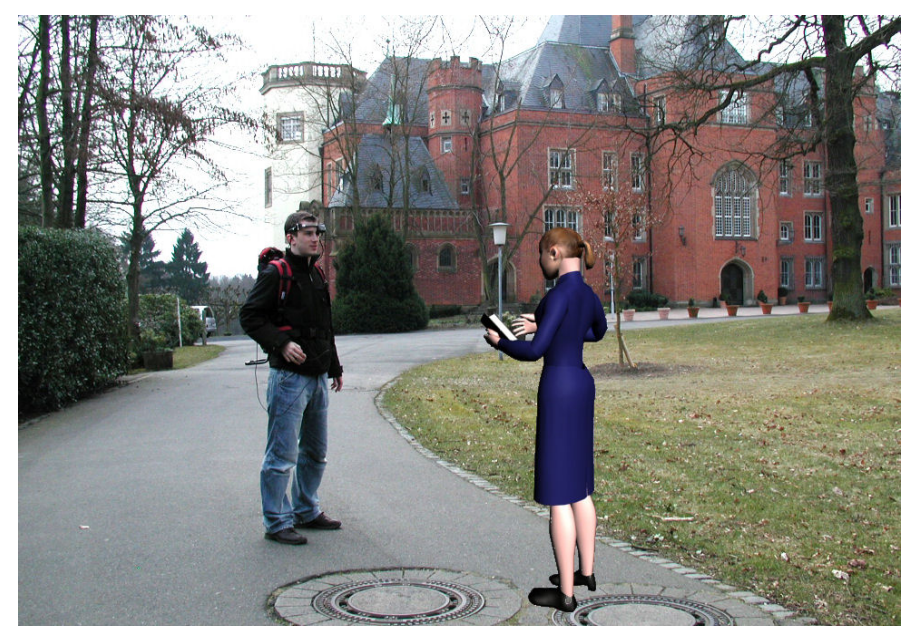

Fig. 1. A virtual anthropomorphic assistant in a mobile environment

The work presented in this paper aims to overcome these shortcomings without the use of complex user interfaces. Interacting with a virtual anthropomorphic character should in theory be similar to communicating with a real assistant (or person), as these types of interface present information in a very natural way. To foster this experience they have to be part of the real world, i.e. by augmenting the real environment rather than being an autonomous interface element on a display. Mobile Augmented Reality (AR) enables us to use virtual assistants as part of the user's local environment almost anywhere and anytime (see figure 1).

In this paper we describe the development of an intuitive multimodal user interface that combines AR technology with a mobile personal assistant. The second part of the paper provides a brief overview of related work, pointing where our approach goes beyond the current state-of-the-art. Section 3 presents our approach in detail, including the hardware setup, the development of the virtual assistant, the environment model and the user interface. In section 4 we describe and discuss the results of our initial field trials. Section 5 provides conclusions and future directions.

\section{Related Work}

In contrast with previous work which either developed an AR information and navigation system or an anthropomorphic assistant, the work presented here concentrates specifically on combining these two technologies.

Mobile augmented reality systems such as the Touring Machine [6] and the applications developed within the OCAR project [11] provide information about the mobile environment by superimposing text into the user's view. The user interfaces of these systems are mostly based on two-dimensional GUIs operated by touchpads, which according to our experience are often considered as somewhat awkward and rather difficult to use. Furthermore, reading requires the user's complete attention. 
Augmented reality systems make use of virtual characters for digital storytelling throughout a city [8] and for guiding school children to outdoor locations with virtual learning content [12]. However, when the user requires additional information and further interactivity the situation becomes more complex. Virtual assistants have mainly been used in virtual reality environments so far. Virtual assistants are in most cases restricted to one specific task. The anthropomorphic assistant Hamilton [13] was designed to arrange furniture in a virtual environment. MAX [9] demonstrates how to assemble a construction kit. Both assistants understand voice commands and interpret hand gestures. Within the research project Virtual Human [16] an interactive learning application was developed: two anthropomorphic characters (a teacher and student) explain requested content in a new and interesting manner.

Welbo [1] was the first anthropomorphic assistant in AR and took the form of a little robot that floats around a living room - the robot was visible to those wearing the HMD. Welbo points at virtual objects and rearranges them upon voice commands. Other virtual characters functioned as game characters [3], test subjects for object design [2] and behavior-driven presenters [4]. However, an anthropomorphic assistant that fulfils tasks for everyday life, as our assistant does, has not yet been developed.

Most AR systems work with small manually entered data sets. Therefore, only little research has been done in the field of databases for mobile AR applications or in the use of environment models and the management of such systems. However as the trend towards location-aware services and assistants grows there is a need for research into storing location based information. The Nexus project [7] explores the creation, definition and development of environment models from heterogenic data sources.

The problem with many of the environment models created so far is that they often only contain geometric and physical data, so data about meanings etc. has to be added manually using keywords. In contrast, as our work is focused on the development of an anthropomorphic assistant the environmental model contains information about the objects and how they can be presented.

\section{Our Approach}

We decided to combine personal information management (PIM) functions with the functionality of a local guide to demonstrate how a mobile user can benefit from an omnipresent anthropomorphic assistant. The approach includes the following five major components, which will be introduced in detail later: the hardware setup, the virtual character, our developed environment model, the software system, and the multimodal user interface.

\subsection{The Mobile AR System}

Our mobile AR setup uses a notebook computer with a $2 \mathrm{GHz}$ processor and an ATI Mobility FireGL V3100 graphics accelerator operating under Windows XP. In outdoor settings, the user's position is tracked by a Holux GPSlim236 GPS receiver. For the time being we abandoned the option of using differential GPS or hybrid outdoor position tracking. For orientation tracking an InterSense InertiaCube 3 is used. 
Indoors, the user's position and orientation is tracked by a combination of ARToolKit tracking and the InertiaCube3. For accessing the tracking devices and for combining the provided data into stable 6-DOF tracking information we use the MORGAN [10] AR/VR framework, which also provides support for the seamless transition between multiple tracking systems (e.g. outdoor and indoor tracking). By doing this the user can roam freely between indoor and outdoor environments. The notebook computer and the GPS receiver are mounted on a backpack worn by the user.

The virtual assistant is superimposed onto the user's view by using a Shimadzu DataGlass 2/A display, this is a monoscopic optical see-through head-worn display. For safety reasons (especially regarding the use in the outdoor environment), we decided for a monoscopic optical see-through display. For head tracking, the InertiaCube 3 and an external active GPS antenna are mounted to the sides of the display. For indoor use - which requires the tracking of the ARToolKit markers - an additional camera is mounted to the display. For speech input and output (from the assistant) we chose a one-ear headset, to reduce magnetic interferences. The alternative input device, a common PC wheel-mouse connected with the laptop computer via BlueTooth, is either carried in one hand or in a (jacket) pocket.

Unlike most other mobile AR configurations, our setup works perfectly without a helmet. For the hardware designated to be head-worn components were chosen that are light-weight and small in size. Moreover, putting on and taking off the modified display and the one-ear headset is very easy. This layout is a prototype and we expect to make some modifications over time so that it is more suitable for daily use.

\subsection{The Virtual Character}

For the rendering of the anthropomorphic character, we extended the MORGAN framework by the open source character animation library Cal3D [14]. As the anthropomorphic assistant places or removes objects from her pockets it was necessary to develop a system that enables her to take out objects like a calendar (for scheduling appointments), a notebook (for writing down notes) and a pen. Further, our software component facilitates the scheduling of all the described animation functions, thus allowing the creation of whole sequences of animations.

Further, in order to equip the assistant with a natural voice, we extended our framework with a component for speech synthesis and output based on Loquendo TTS. The virtual assistant seems more alive to the user and becomes human-like, i.e. anthropomorphic. This impression is further improved by the synchronization of speech and gestures. The assistant appears more acceptable and trustworthy to the user, thus reducing any inhibitions they have when it comes to interacting with it.

The assistant is equipped with elementary movements like walking or running in any desired speed and can nod, shake the head and point into specific directions. During the presentation of objects specifically designed animations are executed, synchronized with speech output. However, to ensure flexibility, the synchronization of presentations cannot be controlled by the application. Rather, it has to be specified in a modifiable database where all information about the world is defined. 


\subsection{An Environment Model}

For the virtual assistant to be a local guide for the user, extensive information about the real world, i.e. the user's current environment, is required. We developed a lightweight, yet powerful environment model. It is based on the widespread XML format GPX [15]. GPX defines waypoints, routes and tracks. We extended the waypoints to utilize them as local environment models.

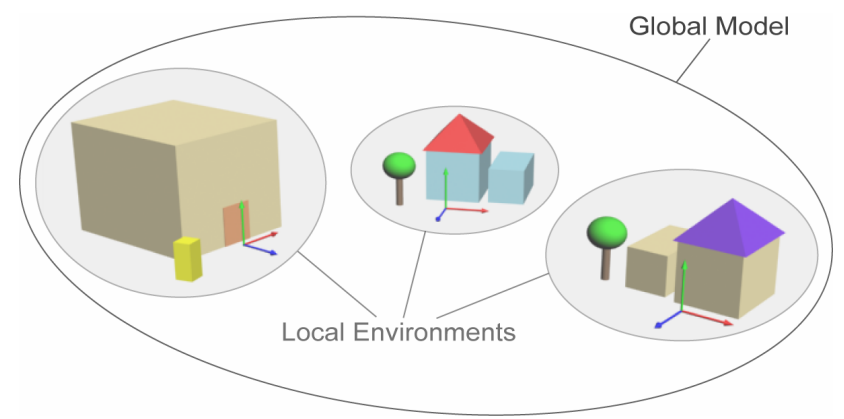

Fig. 2. The sum of all local environments builds a global environment model

An augmented waypoint, i.e. a local environment, is defined as a limited area that can contain objects. Every local environment has its own Cartesian coordinate system in which the contained objects are transformed. Figure 2 illustrates this concept. Besides this transformation, a name, an elevation, a description, a comment, and a type an augmented waypoint provides the following additional information:

- the extent of each object in the local environment: a VRML scene

- a name, a comment, and a description of each contained object

- position and orientation of ARToolKit markers used for indoor tracking

- specific information for the assistant (information about actions to execute for presenting objects or the waypoint itself, data for synchronizing speech and gestures, position to take in for presentations).

We developed an XML schema for an augmented waypoint. An object's information is linked with its 3D data through the unique VRML identifier of the object. The assistant presents an object by reading out aloud its description. Waypoint-specific animations are defined as actions. For the synchronization of gestures and speech, the appropriate action is started when the speech output reaches a corresponding label, which is embedded in the text. For navigation tasks the assistant gets information about the structure of pathways in the user's environment in form of GPX routes.

For the purpose of an anthropomorphic assistant describing and presenting objects to the user, an environment model based on a complex object hierarchy (as found in [7] or [11]) is not needed. When presenting an object by reading a pertinent text, metadata about the object is not needed. Furthermore, since the environment of a mobile user changes continuously, we considered the use of flexible multiple VRML scenes instead of one large global 3D model more appropriate. 


\subsection{The Software System}

Figure 3 gives an overview of the components running within the MORGAN framework. The MARA application controls the assistant, manages the user's personal information and handles all aspects of interaction, while the MORGAN Viewer's purpose is to render the scene graph including the virtual character.

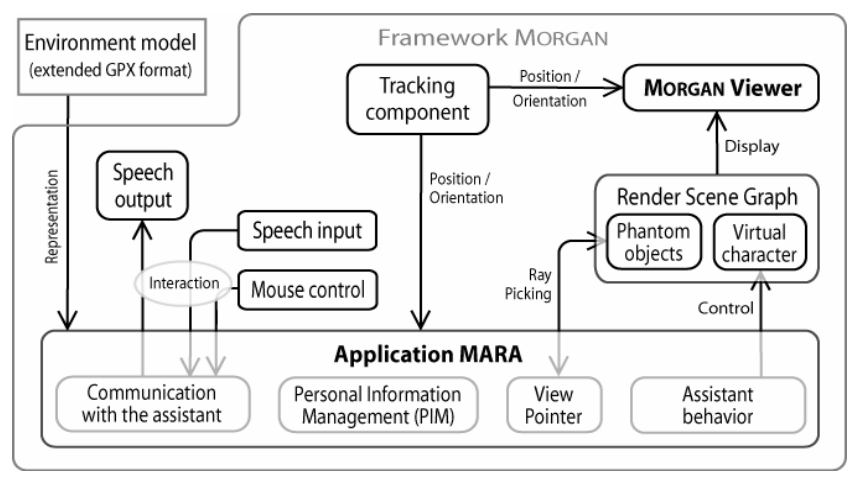

Fig. 3. The modular software system

The tracking component provides continuously updated information about the user's position and viewing direction. The viewer uses this information to set the view point for rendering, the application MARA keeps the assistant informed about the user's whereabouts. The assistant is therefore able to move towards the user while talking to him, to follow him and to turn to him while presenting an object. Using the information about the user's viewing direction we realized a view pointer, allowing the user to select objects simply by gazing at them. For the personal information management (PIM) functionality of the system, the internal database comprises a set of appointments and an ordered list of notes. Appointments can be associated with locations, i.e. with waypoints. The assistant is able to guide the user to an appointment at a desired time, simply by walking ahead. For the navigation a route is planned using paths defined in the environment model.

The MARA application also controls the assistant's behavior. We realized it as a 2-level hierarchical state machine. The higher level states represent the implemented functions:

\section{\begin{tabular}{l|l|l|l|l|l|} 
Idling & Calendar & Notebook & Introduction & Presentation & Guidance \\
\hline
\end{tabular}}

The lower level states correspond to the several steps of the particular functions; e.g. scheduling an appointment requires the specification of a time, a reminder, and an optional location in succession. The assistant displays the current state with distinct gestures and tells the user about ongoing actions. In general, transitions between states are expressed by single animations, e.g. pulling out or putting away a calendar. Figures 4 and 5 provide examples on how the behavior and the current state are projected by the anthropomorphic assistant. Upon receiving a help command the assistant informs the user about the current state and any inputs which may be 
expected from the user. State transitions are mostly caused by user actions, but also by the expiration of time intervals or the end of ongoing actions of the assistant.

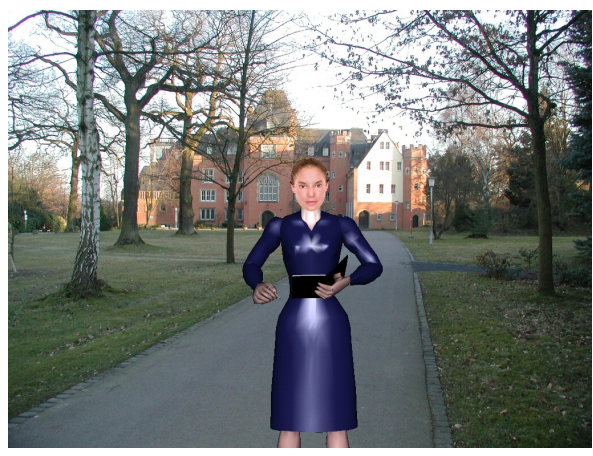

Fig. 4. The virtual assistant with the calendar waiting for input

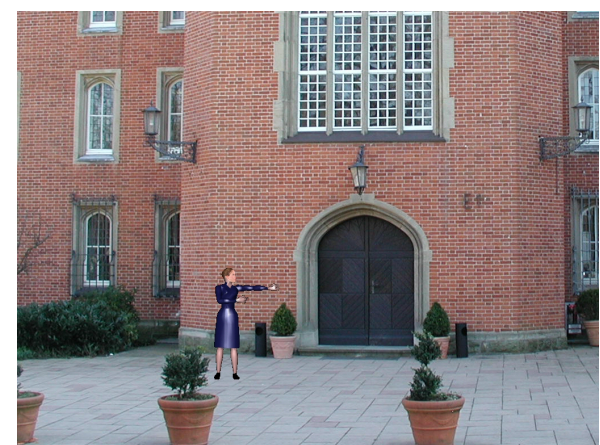

Fig. 5. The virtual assistant presenting the portal of a castle

\subsection{Multimodal Interaction}

To control the system, i.e. to interact with the assistant, two distinct types of interaction are needed:

- communicating with the anthropomorphic assistant

- selecting (real world) objects

As a consequence, the interaction is inherently multimodal. We decided to use speech input as the main user interface for communicating with the assistant. This approach comes closest to communicating with a real person and thus promises a high user acceptance, allowing the user to converse with the virtual assistant.

Our speech input component uses IBM's ViaVoice speech recognition software to transform spoken words, phrases and continuous text into written text and commands. To increase recognition rates we used multiple grammars, one for each function. The user can quickly cancel the current function and change to another mode by issuing short commands. Different phrases with the same meaning can be associated with the same annotation. The system switches automatically between the grammars at highlevel state transitions. The listing in figure 6 shows the intuitive character of the interaction with the assistant.

Situations may occur, in which the user is not able to or does not want to use speech interaction. For these situations, we developed a secondary user interface, utilizing an ordinary PC wheel-mouse. To keep the interaction intuitive, the left mouse button is always used for confirmation or for giving an affirmative answer, while the right mouse button always stops or cancels the current action, dismisses a selection, or negates a question. The wheel is used to change the selection or alter the value that is currently of interest. To keep the user interface as natural as possible, the mouse interaction is designed to function without an on-screen menu. Mouse control and speech input are both usable at the same time, and the user does not need to switch modes. The entire functionality is available through both input methods, with one solitary exception - dictating notes. 


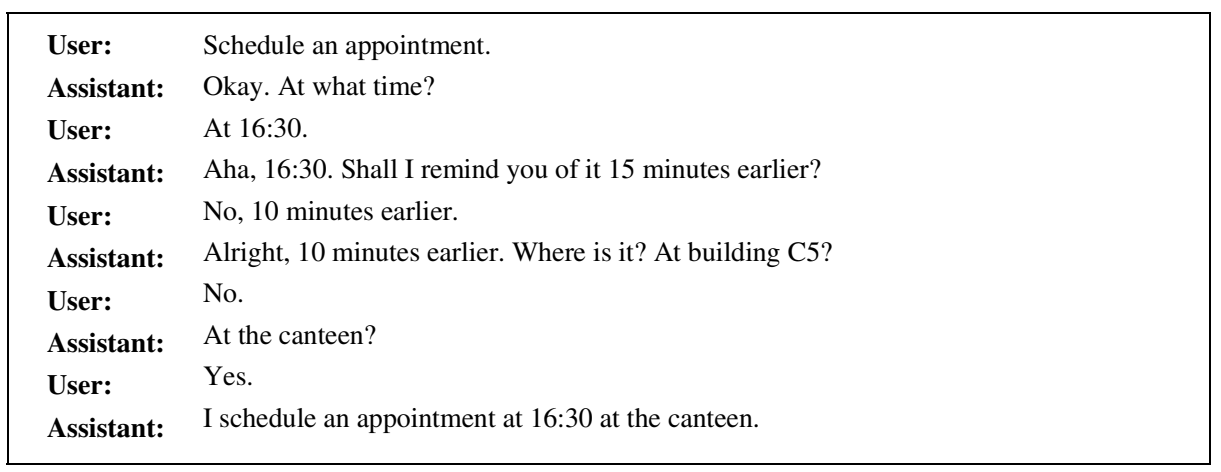

Fig. 6. Dialog between user and assistant

The user selects an object by simply gazing at it for two seconds. The assistant then utters the name of this object and points at it. The help function is accessible at any time, either by saying "help" or by pressing the middle mouse button. The assistant tells about the current state or action and gives interaction hints.

\section{Evaluation}

We evaluated the system in an outdoor setting at our campus. The assistant had information about several waypoints and paths between them. Some of the augmented waypoints contained objects that were selectable (and presentable) separately.

Evaluation Procedure. The evaluation focused on both the functionality of the system and the interaction methods used. The test persons had to make appointments, take notes, let the assistant repeat the notes, make the assistant describe an object or a waypoint and guide the user to an appointment. In order to evaluate the speech interface against mouse input the subjects had to undertake all functions once by speech interaction and once by mouse control. The second part of the evaluation was the completion of a questionnaire. The subjects were asked to rate individual aspects of the user interface as well as the sustainability of the overall approach.

Test Group. 13 volunteers participated in the evaluation ( 7 male, 6 female). The age varied between 16 and 45. Nine participants were frequent computer users, also nine participants said they used the PIM functionality of their mobile phones, five were frequent PDA users, and four indicated they used in-car navigation system.

Results. In order to ensure a fair study the speech recognition system was not trained to recognize any specific voice. Nonetheless, recognition rates varied strongly from user to user. As would be expected the subjects rating of the speech interface was heavily influenced by the number of recognition errors. The mouse as an alternate input method was considered rather feasible. One important finding is that the amount of time taken to complete a task was similar for both input methods. Also, the view pointer for the selection of objects was generally considered intuitive. 


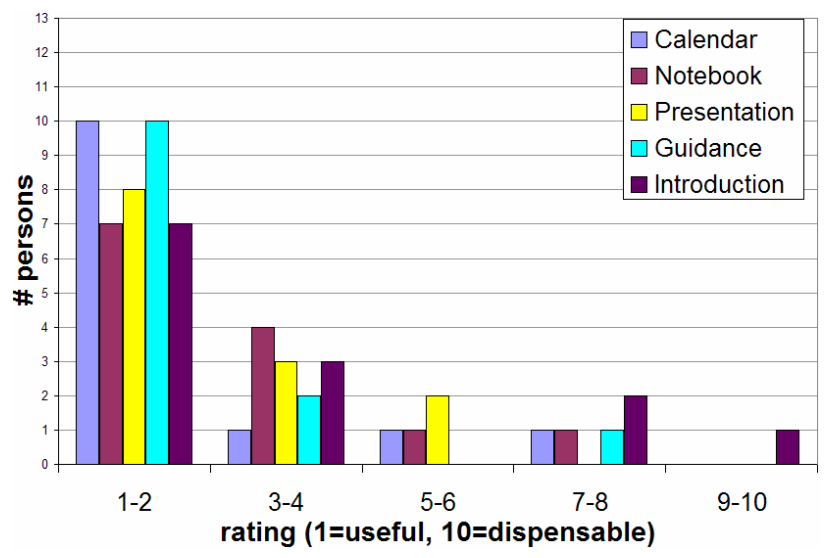

Fig. 7. Rating of the implemented functions

As figure 7 illustrates, the selection of the functions implemented in the system was found to be useful. The users also liked the idea of the assistant being both a secretary and a local guide. All test persons appreciated the help function and considered it user friendly.

About half of the users declared that the assistant's state and behavior were not always clear. That was mostly due to the limited field of view (FOV) of the display and its sensitivity to bright daylight. The assistant could only be seen in a small area and was, due to contrast issues, not always visible in bright daylight. As a result, only half of the users accepted the assistant as a proper substitute for a human assistant. The other half were pleased with the speech (and mouse) interface and considered the virtual character a side-product. Also, some subjects stated that they would appreciate more visual and acoustic feedback from the assistant and eventually visual head-up information, apart from the virtual character.

The study points to people liking the idea of an anthropomorphic assistant; however it also points to there being a need to improve speech recognition, the display and to make the system more wearable.

\section{Conclusions and Future Work}

In this paper we introduced MARA - our omnipresent personal virtual assistant, representing a first step towards integrating PIM functionality with services that are usually offered by personal guides and secretaries. We developed this by combining mobile AR technology, an anthropomorphic user interface representation in the local environment of the user, an intuitive multi-modal user interface, and a location-aware application. We evaluated our approach in an outdoor setting. Based on our findings we would expect that such a system would enjoy wide user acceptance. Anthropomorphic characters in mobile AR systems however require significant improvements in underlying technologies (especially with the quality of the headworn displays) in order to form a good alternative to more traditional user interfaces.

Our intention is to modify the system to take into account the findings of the evaluation. This includes supporting additional awareness and feedback mechanisms as 
well as making the system more flexible and scalable (as suggested in [5]). Next steps include the integration of online communication (email, VoIP). Additionally, in a further evaluation we will compare the anthropomorphic character with other interface options.

Acknowledgments. The authors thank their colleagues at Fraunhofer FIT, especially Jan Ohlenburg, for their support and contributions regarding the implementation of MARA. Further thanks belong to all volunteers participating in the evaluation.

\section{References}

1. Anabuki, M., Kabuka, H., Yamamoto, H., Tamura, H.: Welbo: An Embodied Conversational Agent Living in Mixed Reality Space. CHI', extended abstracts, pp. 10-11 (2000)

2. Balcisoy, S., Kallmann, M., Torre, R., Fua, P., Thalmann, D.: Interaction Techniques with Virtual Humans in Mixed Environments. Proc. of International Symposium on Mixed Reality, Yokohama, Japan (2001)

3. Barakonyi, I., Weilguny, M., Psik, T., Schmalstieg, D.: MonkeyBridge: Autonomous Agents in Augmented Reality Games. In: Proc. of ACM SIGCHI International Conference on Advances in Computer Entertainment Technology (ACE’05), Valencia, Spain (2005)

4. Barakonyi, I., Schmalstieg, D.: Ubiquitous Animated Agents for Augmented Reality. In: Proc. of ISMAR'06, Santa Barbara, CA, USA (2006)

5. Broll, W., Schäfer, L., Höllerer, T., Bowman, D.: Interface with Angels: The Future of VR and AR Interfaces. IEEE Computer Graphics and Applications 21(6), 14-17 (2001)

6. Feiner, S., MacIntyre, B., Höllerer, T., Webster, A.: A Touring Machine: Prototyping 3D Mobile Augmented Reality Systems for Exploring the Urban Environment. In: Proc. ISCW '97, pp. 74-81, Cambridge, MA, USA (1997)

7. Hohl, F., Kubach, U., Leonhardi, A., Rothermel, K., Schwehm, M.: Nexus - An Open Global Infrastructure for Spatial-Aware Applications. In: Proc. of MobiCom '99, Seattle, WA, USA (1999)

8. Holweg, D., Schneider, O.: GEIST - Mobile Outdoor AR-Informationsystem for historical education with digital storytelling. BMBF Virtual Reality Status Conference, (2004)

9. Kopp, S., Jung, B., Leßmann, N., Wachsmuth, I.: Max - A Multimodal Assistant in Virtual Reality Construction. KI - Künstliche Intelligenz 4/03, special Issue on Embodied Conversational Agents. Bremen, Germany, pp. 11-17 (2003)

10. Ohlenburg, J., Herbst, I., Lindt, I., Fröhlich, T., Broll, W.: The MORGAN Framework: Enabling Dynamic Multi-User AR and VR Projects. In: Proc. of VRST'04, pp. 166-169 (2004)

11. Reitmayr, G., Schmalstieg, D.: Collaborative Augmented Reality for Outdoor Navigation and Information Browsing. In: Proc. of Symposium Location Based Services and TeleCartography 66, 53-59 (2004)

12. Sephton, T.: Teaching agents for wearable augmented reality systems. In: Fensel, D., Sycara, K.P., Mylopoulos, J. (eds.) ISWC 2003. LNCS, vol. 2870, pp. 250-251. Springer, Heidelberg (2003)

13. Wachsmuth, I., Lenzmann, B., Jörding, T., Jung, B., Latoschik, M., Fröhlich, M.: A virtual interface agent and its agency. In: Proc. of the First International Conference on Autonomous Agents, pp. 516-517. ACM Press, New York (1997)

14. Cal3D website: http://cal3d.sourceforge.net

15. GPX website: http://www.topografix.com/gpx

16. Virtual Human website: http://www.virtual-human.org 